\title{
Multiple Adaptive Mechanisms to Chronic Liver Disease Revealed at Early Stages of Liver Carcinogenesis in the Mdr2-Knockout Mice
}

\author{
Mark Katzenellenbogen,' Orit Pappo, ${ }^{2}$ Hila Barash, ${ }^{1}$ Naama Klopstock, ${ }^{1}$ Lina Mizrahi, \\ Devorah Olam, Jasmine Jacob-Hirsch, ${ }^{4}$ Ninette Amariglio, ${ }^{4}$ Gidi Rechavi, ${ }^{4}$ \\ Leslie Ann Mitchell, ${ }^{1}$ Ron Kohen, ${ }^{3}$ Eytan Domany, ${ }^{5}$ \\ Eithan Galun, ${ }^{1}$ and Daniel Goldenberg ${ }^{1}$
}

${ }^{1}$ Goldyne Savad Institute of Gene Therapy and ${ }^{2}$ Department of Pathology, Hadassah University Hospital; ${ }^{3}$ Department of Pharmacology, School of Pharmacy, The Hebrew University of Jerusalem, Jerusalem, Israel; ${ }^{4}$ Pediatric Hemato-Oncology Department and Institute of Hematology, Chaim Sheba Medical Center, Tel-Hashomer, Tel-Aviv University, Tel-Aviv, Israel; and

${ }^{5}$ Department of Physics of Complex Systems, Weizmann Institute of Science, Rehovot, Israel

\begin{abstract}
Molecular events preceding the development of hepatocellular carcinoma were studied in the Mdr2-knockout (Mdr2-KO) mice. These mice lack the liver-specific P-glycoprotein responsible for phosphatidylcholine transport across the canalicular membrane. Portal inflammation ensues at an early age followed by hepatocellular carcinoma development after the age of 1 year. Liver tissue samples of Mdr2-KO mice in the early and late precancerous stages of liver disease were subjected to histologic, biochemical, and gene expression profiling analysis. In an early stage, multiple protective mechanisms were found, including induction of many antiinflammatory and antioxidant genes and increase of total antioxidant capacity of liver tissue. Despite stimulation of hepatocyte DNA replication, their mitotic activity was blocked at this stage. In the late stage of the disease, although the total antioxidant capacity of liver tissue of Mdr2-KO mice was normal, and inflammation was less prominent, many protective genes remained overexpressed. Increased mitotic activity of hepatocytes resulted in multiple dysplastic nodules, some of them being steatotic. Expression of many genes regulating lipid and phospholipid metabolism was distorted, including up-regulation of choline kinase A, a known oncogene. Many other oncogenes, including cyclin D1, Jun, and some Ras homologues, were up-regulated in Mdr2-KO mice at both stages of liver disease. However, we found no increase of Ras activation. Our data suggest that some of the adaptive mechanisms induced in the early stages of hepatic disease, which protect the liver from injury, could have an effect in hepatocarcinogenesis at later stages of the disease in this hepatocellular carcinoma model. (Cancer Res 2006; 66(8): 4001-10)
\end{abstract}

\section{Introduction}

Hepatocarcinogenesis has many features that are common in mice and humans. Various mouse models of hepatocellular

Note: Supplementary data for this article are available at Cancer Research Online (http://cancerres.aacrjournals.org/).

E. Galun is the Sam and Ellie Fishman Chair in Gene Therapy.

E. Domany is the incumbent of the Henry J. Leir Professorial Chair.

Requests for reprints: Daniel Goldenberg, Goldyne Savad Institute of Gene Therapy, Hadassah University Hospital, P.O. Box 12000, Jerusalem 91120, Israel. Phone: 972-2-677-8108; Fax: 972-2-643-0982; E-mail: goldenberg@hadassah.org.il.

C 2006 American Association for Cancer Research.

doi:10.1158/0008-5472.CAN-05-2937 carcinoma, including knockout and transgenic animals, have been used to study the molecular mechanisms of liver cancer. Comparative analysis of global gene expression patterns of different mouse hepatocellular carcinoma models and human hepatocellular carcinoma samples showed that most mouse hepatocellular carcinomas are similar to specific subgroups of human hepatocellular carcinomas and thus may be useful in modeling studies (1).

To study the molecular mechanisms of hepatocellular carcinoma development and to find candidate genes for gene therapy intervention in the early stages of the disease, we did intensive analysis of liver tissues of the Mdr2-knockout (Mdr2-KO) mice in the precancerous stages of liver disease. These mice lack the liver-specific P-glycoprotein responsible for phosphatidylcholine transport across the canalicular membrane (2). The absence of phospholipids from bile leads to bile regurgitation into the portal tracts (3), causing portal inflammation that ensues at an early age followed by slowly developing hepatocellular carcinoma, which, in this regard, mimics the human disease (4).

In the present study, we investigated different signaling pathways associated with precancerous stages of liver disease in the Mdr2KO model. Liver tissue samples of Mdr2-KO and control Mdr2heterozygous mice at ages 3 and 12 months were subjected to histologic, biochemical, and gene expression profiling analysis. Results revealed complex interactions between signaling pathways that control inflammatory reactions, response to oxidative stress, lipid metabolism, and cell proliferation. These findings suggest that induction of multiple adaptive mechanisms protecting liver tissue in the early stages of the disease as well as progressively disturbed lipid and phospholipid metabolism may have an effect in hepatocellular carcinoma development in the later stages of liver disease in Mdr2-KO mice.

\section{Materials and Methods}

Mice. Founders of the FVB.129P2-Abcb4 ${ }^{\text {tm1Bor }}$ (Mdr2-KO; old name FVB.129P2-Pgy $2^{\mathrm{tm} 1 \mathrm{Bor}}$ ) and the wild-type $\mathrm{FVB} / \mathrm{NJ}$ mice were purchased from The Jackson Laboratory (Bar Harbor, ME). Colonies of both strains were maintained under specific pathogen-free conditions in the Animal Facility of The Hebrew University Medical School. The $\mathrm{F}_{1}$ hybrids produced by breeding of a FVB.129P2-Abcb $4^{\text {tm1Bor }}$ male and a $\mathrm{FVB} / \mathrm{NJ}$ female were used as age-matched controls.

Harvesting of mouse liver tissue. Nonfasting male Mdr2-KO or control heterozygous mice were anesthetized with isoflurane and sacrificed by cervical dislocation. Livers were rapidly excised and weighed; part of the left lobe was fixed in $4 \%$ buffered formaldehyde for histologic analysis, and the remaining liver tissue was quickly frozen in liquid nitrogen and stored at 
$-80^{\circ} \mathrm{C}$ until use. All mice were operated at the same time interval between 12:00 a.m. and 15:00 p.m.

Blood analysis. Blood was collected by orbital bleeding. Levels of liver enzymes in sera were determined using Reflotron (Roche, Mannheim, Germany).

Gene expression profiling. Total RNA was isolated from frozen liver tissues with Trizol reagent (Invitrogen, Carlsbad, CA) as described by the manufacturer. Gene expression profiling was done using Affymetrix (Santa Clara, CA) Mouse Genome Array 430A ( 22,000 genes). Data were analyzed using Microarray Analysis Suite version 5 (MAS 5.0; Affymetrix), Matlab software (MathWorks, Natick, MA), and superparamagnetic (SPC) clustering algorithm (5) as described in Supplementary Data.

Deletions in mitochondrial DNA. The D1 deletion in mitochondrial DNA (mtDNA), which accumulates in livers of aging mice, was detected by semiquantitative reverse transcription-PCR (RT-PCR) using primers "mtD1" flanking the deletion ends (Supplementary Table S1) and total liver DNA as a template as described in ref. 6. A stable fragment of mtDNA that does not produce deletions with age was used as control (primers "mtDNA").

Protein oxidation. The degree of protein oxidation in liver tissue was evaluated using the "Oxyblot" kit (Chemicon, Pittsburgh, PA) as recommended by the manufacturer. The method is based on immunodetection of carbonyl groups that are a hallmark of the oxidative status of proteins.

Total antioxidant capacity. The total antioxidant capacity of liver tissues was evaluated by modifying the method described by Kawagoe and Nakagawa (7). Total antioxidant capacity of liver tissue is inversely proportional to its $\mathrm{IC}_{50}$, which was estimated by adding to serial dilutions of liver extracts the hydrophilic free radical initiator 2,2'azobis(2-amidinopropane) dihydrochloride (AAPH; Wako Chemicals, Neuss, Germany), and luminol as a chemiluminescence amplifier. Percentage of inhibition for each dilution was calculated as the decrease of luminescence from that of the most diluted sample. The data were normalized to protein concentration measured by the Bradford method. The $\mathrm{IC}_{50}$ was defined based on polynomial regression of the inhibitory curves. Chemiluminescence was measured by a "Mithras LB 940" luminometer (Berthold Technologies, Bad Wildbad, Germany).

Ras activation assay. Detection of Ras-GTP complexes by pull-down assay with glutathione $S$-transferase (GST)-Rafl-RBD protein was done using the EZ-Detect Ras activation kit (Pierce, Rockford, IL) as described by the manufacturer.

Statistical analysis. The total antioxidant capacity of liver tissue, the liver-to-body weight relation, and the liver mitotic activity were evaluated with the two-tailed $t$ test. The age-associated changes in serum concentration of liver enzymes were evaluated with the one-tailed $t$ test.

\section{Results}

Characterization of Mdr2-KO mice. We used the Mdr2-KO strain FVB.129P2-Pgy $2^{\text {tmlBor }}$ (4), which develops liver tumors at age 15 months with $\sim 100 \%$ efficiency. ${ }^{6}$ As a control, we used its hybrid with the parental strain $\mathrm{FVB} / \mathrm{N}$ ( $\mathrm{F}_{1}$ generation only). Although these hybrid heterozygotes $\operatorname{Mdr} 2(+/-)$ had a genotype highly similar to that of Mdr2-KO, they were phenotypically healthy and did not develop liver tumors over the 2-year monitoring period. To follow developmental differences in the precancerous stages, we studied liver tissue and sera from Mdr2-KO and control Mdr2(+/-) males at ages 3, 6, 9, and 12 months. The Mdr2-KO strain showed significantly elevated serum levels of liver enzymes (Fig. $1 A$ ) and mild hepatomegaly (Fig. $1 B$ ) during all precancerous disease stages. Hepatocyte proliferation in Mdr2-KO mice increased dramatically between age 6 and 9 months (Fig. $1 C$ ). At the age of 12 months,

\footnotetext{
${ }^{6}$ M. Katzenellenbogen et al., in preparation.
}

most Mdr2-KO mouse livers had one or two visible steatotic nodules measuring 1 to $3 \mathrm{~mm}$ in diameter (Fig. $2 A$ ).

Analysis of gene expression profiling. RNA samples from livers of Mdr2-KO and control heterozygous 3- and 12-month-old mice (three males per experimental group) were subjected to genome-scale gene expression profiling. The gene expression values were extracted with MAS 5.0 software and analyzed by cluster analysis, fold change filtering, and statistical tests. The cluster analysis of the expression data resulted in division of samples according to the experimental groups (Fig. $3 A$ ). The SPC cluster analysis $(5,8)$ of genes applied alone or in combination with gene prefiltering, based on differential expression between the experimental groups, revealed several gene clusters characterized by common expression patterns and similar functions. Clusters containing genes, which were progressively up-regulated from ages 3 to 12 months, were enriched with genes involved in cell proliferation.

Evaluation of differential expression of genes between mutants and heterozygotes of both ages using fold change filters (the 2- or 1.8-fold difference) showed a significant decrease in the number of differentially expressed genes from ages 3 to 12 months. The difference was more dramatic for down-regulated genes (Fig. $3 B$ ). Functional categories of genes with at least 2-fold differential expression between mutants and controls are shown in Table 1. Statistical analysis of Gene Ontology definitions done with EASE online tool revealed that, at both ages, the processes related to immune and inflammatory responses and to DNA replication were the most significant.

Histology and analysis of immune/inflammatory genes and cells. Pathologic scores of H\&E-stained liver sections from 3- and 12-month-old Mdr2-KO mice were significantly higher than those of control mice (Supplementary Table S2). Livers of 3-month-old Mdr2-KO mice had characteristic portal inflammation, portal tract expansion due to bile duct hyperplasia, and fibrosis with early septal formation (Fig. 2B). At 12 months, inflammatory infiltrates in the portal tracts of Mdr2-KO livers were less pronounced (Supplementary Fig. S1B), and the lobular architecture was disrupted, causing difficulty in detecting parenchymal fibrosis. Focal accumulations of inflammatory cells were observed within lobular areas, suggesting ongoing inflammation.

Inflammatory cell infiltrates were further characterized by staining for monocytes/macrophages (F4/80) and neutrophil myeloperoxidase. In livers of 3-month-old mutants, a moderate increase in myeloperoxidase-positive cells was observed in the inflamed portal tracts (data not shown). However, at 3 months, many F4/80-positive cells were observed in the portal tracts of Mdr2-KO mice relative to control mice (Fig. $2 B$ ), suggesting a massive infiltration of monocytes/macrophages. These patterns prevailed in samples taken from 12-month-old mice, although the numbers of inflammatory cells in the portal tracts seemed reduced in comparison with those observed at 3 months (data not shown).

About $10 \%$ of differentially expressed genes detected by Affymetrix microarrays at both ages 3 and 12 months were associated with the immune/inflammatory response (Table 1). In agreement with the immunohistochemistry data, genes typically expressed by monocytes/macrophages [Cd14 and $C d 36$ (Table 2) and Aif1, Cd68, Csf1r, Ctss, Gbp2, Igtp, Irf7, Mpeg1, and Plk3 (Supplementary Table S3)] were overexpressed mostly at 3 months. Differential expression of some of these genes was confirmed by semiquantitative RT-PCR (Supplementary Fig. S2). Of interest was the up-regulation in Mdr2-KO livers of the neutrophil-specific gene 


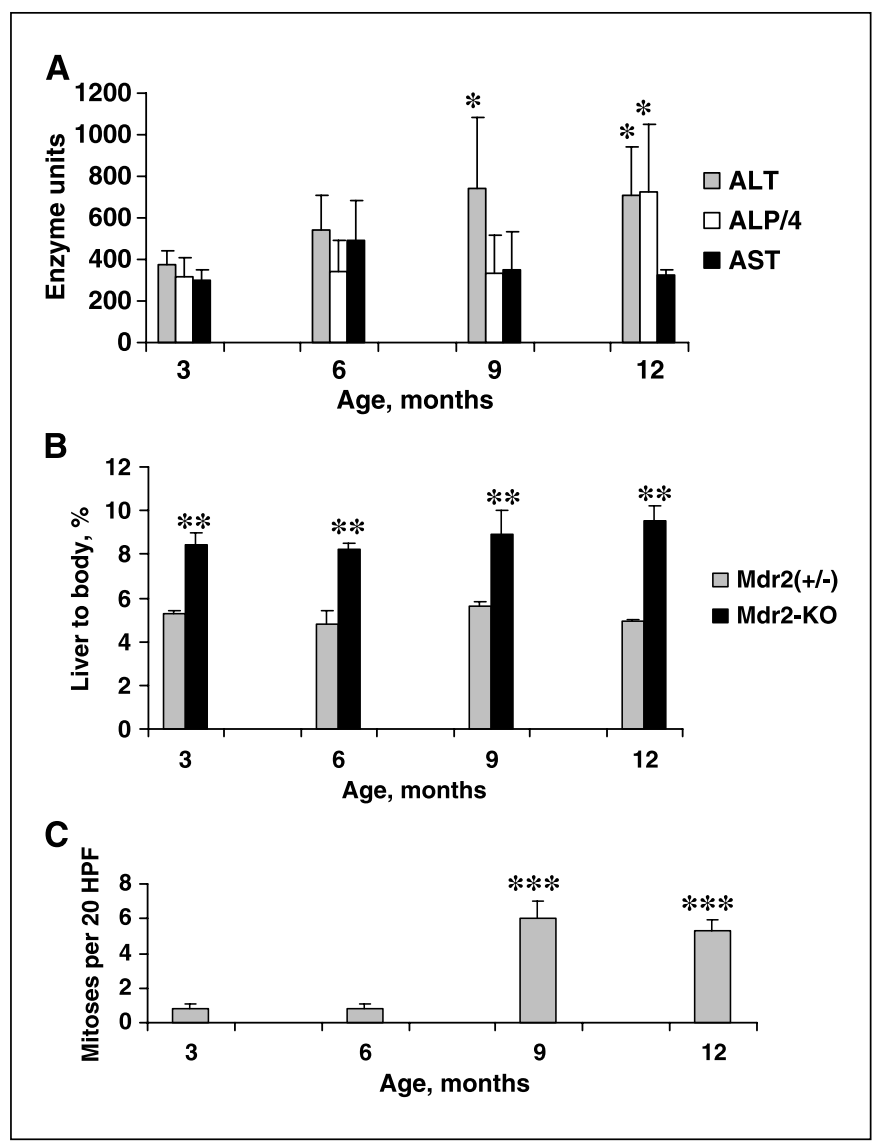

Figure 1. Age-associated physiologic changes in Mdr2-KO mice. $A$, levels of liver enzymes in serum (units). $A L T$, alanine aminotransferase; $A L P / 4$, alkaline phosphatase level divided to 4 (to fit to the scale); $A S T$, aspartate aminotransferase. $B$, liver-to-body weight $(\%) . C$, number of visible hepatocyte mitoses per 20 high-power fields (HPF). Columns, mean of four males; bars, SD. *, $P<0.05$, compared with 3-month-old mice; ${ }^{* *}, P<0.01$, compared with heterozygous control; ${ }^{\star \star \star}, P<0.01$, compared with 3 - and 6 -month-old mice.

Lcn2 and of Saal and Saa2 genes at both ages (Table 2). Genes Saal and Saa2 encode acute-phase reactants, serum amyloid A proteins that may stimulate expression and release of tumor necrosis factor- $\alpha$ (TNF- $\alpha)$ in neutrophils (9) and induce nuclear factor- $\kappa \mathrm{B}(\mathrm{NF}-\kappa \mathrm{B})$ transcriptional activity in epithelial cells (10).

Expression of several important regulators and mediators of immune/inflammatory response, which was not detected by Affymetrix microarray, was tested by semiquantitative RT-PCR. Inducible nitric oxide synthase (gene Nos2), a marker of macrophage activation, was not differentially expressed at either age 3 or 12 months; the same was also true for transforming growth factor- $\beta$ (data not shown). In contrast, the $T N F-\alpha$ gene was overexpressed in the livers of Mdr2-KO mice at both ages (Supplementary Fig. S2).

Among the identified up-regulated genes whose products could potentially be responsible for suppression of macrophage activation were Lgals1, encoding galectin-1 (11); Axl, encoding receptor protein-tyrosine kinase and its ligand Gas6 (12); Pparg, encoding peroxisome proliferator-activated receptor- $\gamma$ (PPAR- $\gamma$; ref. 13); Spp1, encoding osteopontin (14); and Slpi, encoding secretory leukocyte protease inhibitor (15). Overexpression of some of these genes at 3 months was confirmed by semiquantitative RT-PCR (Supplementary Fig. S2A).

The Slpi gene, highly up-regulated in the livers of Mdr2-KO mice at both ages (Table 2), encodes a serine protease inhibitor having multiple anti-inflammatory activities (15). To confirm its overexpression and to determine its cellular localization, we did immunostaining (Supplementary Fig. S3). In Mdr2-KO mice at 3 months, a very strong cytoplasmic staining was observed in hepatocytes located in the centrolobular areas throughout the liver, suggesting a role for Slpi in the control of the acute inflammatory response (Supplementary Fig. S3B).

These results show a clear difference between the two stages of liver disease in Mdr2-KO mice: the acute stage at age 3 months and the chronic stage at age 12 months. In the acute stage, intensive inflammation of portal tracts was associated with massive invasion of macrophages accompanied by neutrophils. During inflammation, several adaptive protective mechanisms seemed to have been activated, including up-regulation of genes whose products may suppress activation of macrophages and neutrophils. Most of these genes were also overexpressed at 12 months, when inflammation was reduced.

Analysis of oxidative stress and antioxidant systems. Gene expression profiling revealed induction of many genes involved in response to oxidative stress in the livers of Mdr2-KO mice especially at age 3 months (Table 2). Some of these genes control the metabolism of glutathione, a principal antioxidant, and included the following: several forms of GST (Gsta), glutathione peroxidase 4 (Gpx4), glutathione synthase (Gss), and one of the main transcriptional activators of antioxidant genes, $\mathrm{Nrf} 2$ (encoded by the $N f e 2 l 2$ gene). Overexpression of Gss and $N f e 2 l 2$ genes at 3 months was confirmed by RT-PCR (Supplementary Fig. S2A). To evaluate the degree of oxidative stress and the total antioxidant capacity in the livers of Mdr2-KO mice, we measured the levels of total liver protein oxidation, frequency of deletions in mtDNA, and ability of liver extracts to inhibit free radical generator-induced chemiluminescence. No significant difference was found in the oxidative levels of total liver proteins between Mdr2-KO and control heterozygous mice at age 12 months, whereas at age 3 months noticeably fewer oxidized proteins were observed in the livers of Mdr2-KO mice (Fig. $4 A$ ).

In parallel, we estimated the frequency of a specific deletion in mtDNA, which normally appears only with aging (6), or due to oxidative stress (16). At age 3 months, the deletion was not detected in either the control heterozygous or Mdr2-KO mice. At 12 months, the deletion was hardly detected in Mdr2-KO mice but was abundant in all control mice (Fig. $4 C$ ). The total level of mtDNA in Mdr2-KO mice was only slightly reduced in comparison with control mice. These data show that in both tested ages there was no evidence of an increased oxidative stress in Mdr2-KO mice.

We further assessed the total antioxidant capacity of liver tissues by measuring its inverse variable, the concentration of liver tissue extract that provided $50 \%$ inhibition of luminol-amplified chemiluminescence initiated by AAPH. The total antioxidant capacity of the liver tissue of 3-month-old Mdr2-KO mice was higher than that of controls; however, this difference was not evident at age 12 months (Fig. $4 B$ ). The first two parts of Fig. 4 are remarkably similar, thus reflecting the inverse relation between the total antioxidant capacity of a tissue and the degree of its total protein oxidation. Similarly, cyclic voltammetry analysis (17) revealed decreased total antioxidant capacity of the liver tissue extract aqueous phase obtained from the 12-month-old Mdr2-KO mice compared with 3-month-old animals (data not shown).

These data show an increased expression of oxidative stress/ antioxidant genes, reduced protein oxidation, and increased total antioxidant capacity of liver tissues in 3-month-old Mdr2-KO mice. 
At 12 months, the only difference between Mdr2-KO and control mice was overexpression of some oxidative stress/antioxidant genes. These observations suggest an induction of protective antioxidant systems in the livers of Mdr2-KO mice at an early age most probably due to a response to oxidative stress caused by defects in bile metabolism and/or by inflammation.

Lipid metabolism. Steatotic foci were observed in the livers of Mdr2-KO mice at age 12 months (Fig. $2 A$ ) but not at age 3, 6, or 9 months. Many genes that control metabolism of lipids and fatty acids were differentially expressed in the livers of Mdr2-KO mice according to microarray data (Tables 1 and 2). Some were upregulated in Mdr2-KO mice at both ages (ApoA4, ApoC2, Cd36, Lpl, Pparg, and Ptgds) and others were only up-regulated at 12 months (Cav1, Cav2, Chka, Fabp4, Fasn, Pdk4, Pld1, Scd1, and Scd2). Gene Ptgds was strongly up-regulated in the livers of Mdr2-KO mice at both ages but more significantly at age 12 months (Table 2). It encodes the key enzyme for production of the proinflammatory prostaglandin $D_{2}$, whose secondary derivative, 15-deoxy- $\Delta 12,14$ prostaglandin $\mathrm{J}_{2}\left(15 \mathrm{~d}-\mathrm{PGJ}_{2}\right)$, has antiproliferative and antiinflammatory activities. Prostaglandin $15 \mathrm{~d}-\mathrm{PGJ}_{2}$ is a natural ligand of the PPAR- $\gamma$ receptor, and some of its physiologic effects are mediated through PPAR- $\gamma$ (18).

Up-regulation of several PPAR- $\gamma$ target genes (Fabp4, Cav1, Cav2, $C d 36, P d k 4, L p l$, and $S c d 1$ ) in the microarray (Table 2) as well as validation of Pparg up-regulation by RT-PCR in 3- and 12-month- old Mdr2-KO mice (Supplementary Fig. S2) support the notion that PPAR- $\gamma$ signaling pathways are activated in Mdr2-KO mice especially at age 12 months. The prosteatotic role of PPAR- $\gamma$ in mouse liver was shown previously by development of liver steatosis in mice that overexpress it, specifically in the liver (19), and by significant reduction of hepatic steatosis in two mouse models with liver-specific deletion of its gene $(20,21)$. Similarly, liver-specific expression of the gene $\mathrm{Lpl}$, encoding lipoprotein lipase, resulted in the uptake of circulating triglycerides and development of hepatosteatosis (22). In general, $L p l$ is not expressed in adult liver; however, it can be induced by TNF- $\alpha$ or by activation of PPAR- $\gamma$ (22). $L p l$ is also activated by apolipoproteins C-II and A-IV, the only two apolipoproteins that were overexpressed in Mdr2-KO mice at both ages (Table 2).

Phosphatidylcholine metabolism. Several genes involved in the regulation of phosphatidylcholine metabolism were differentially expressed in Mdr2-KO mice at different stages of the disease. In 3-month-old Mdr2-KO mice, down-regulation of the last enzyme in phosphatidylcholine biosynthetic pathway (encoded by gene Chpt 1) and up-regulation of one of the first enzymes to degrade phosphatidylcholine (two subunits of phospholipase C, encoded by genes Plce1 and Plcg2) was observed at mRNA level (Table 2). In 12-month-old Mdr2-KO mice, mRNA levels for these enzymes were similar to those in controls; however, gene Pld1, encoding another phosphatidylcholine-degrading enzyme, phospholipase D1,

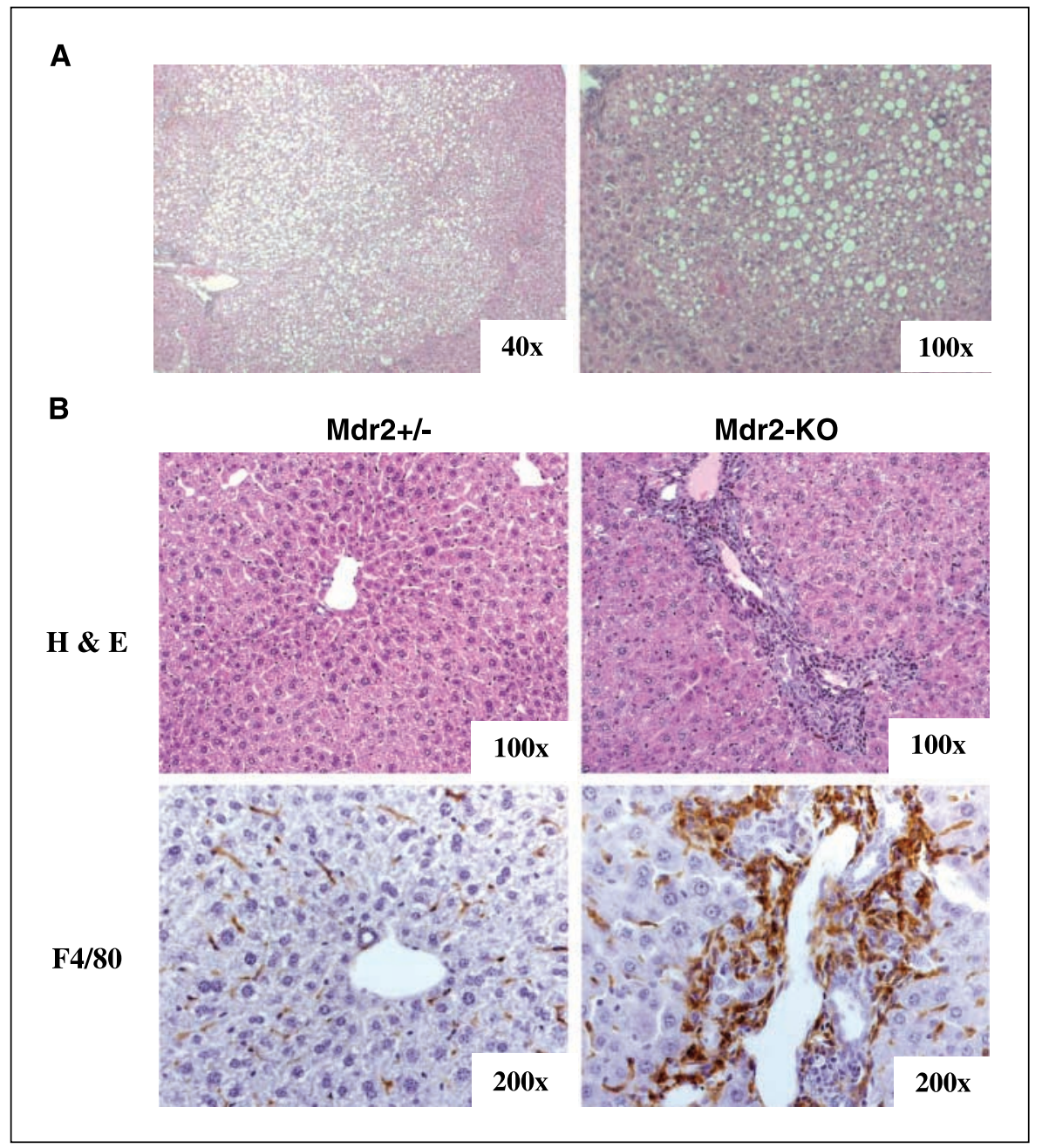

Figure 2. Histologic analysis of the livers of Mdr2-KO and healthy control Mdr2(+/-) mice. $A$, steatotic nodule in the liver of a 12-month-old Mdr2-KO mouse. Magnification, $\times 40$ (left) and $\times 100$ (right). $B$, inflammatory patterns in the livers of Mdr2-KO and control Mdr2(+/-) mice at age 3 months. Top, H\&E-stained sections of liver tissue showing normal portal tract morphology in a control mouse (left) and bile duct proliferation, inflammatory cell infiltration, and fibrosis in the Mdr2-KO mouse (right). Bottom, liver tissue sections stained with $\mathrm{F} 4 / 80$ monocyte/ macrophage-specific antibody showing staining of only Kupffer cells in the liver of a control mouse (left) and intense infiltration of portal tracts and increased numbers of F4/80-positive cells in the liver of a Mdr2-KO mouse (right). Magnification, $\times 100$ (top) and $\times 200$ (bottom). 


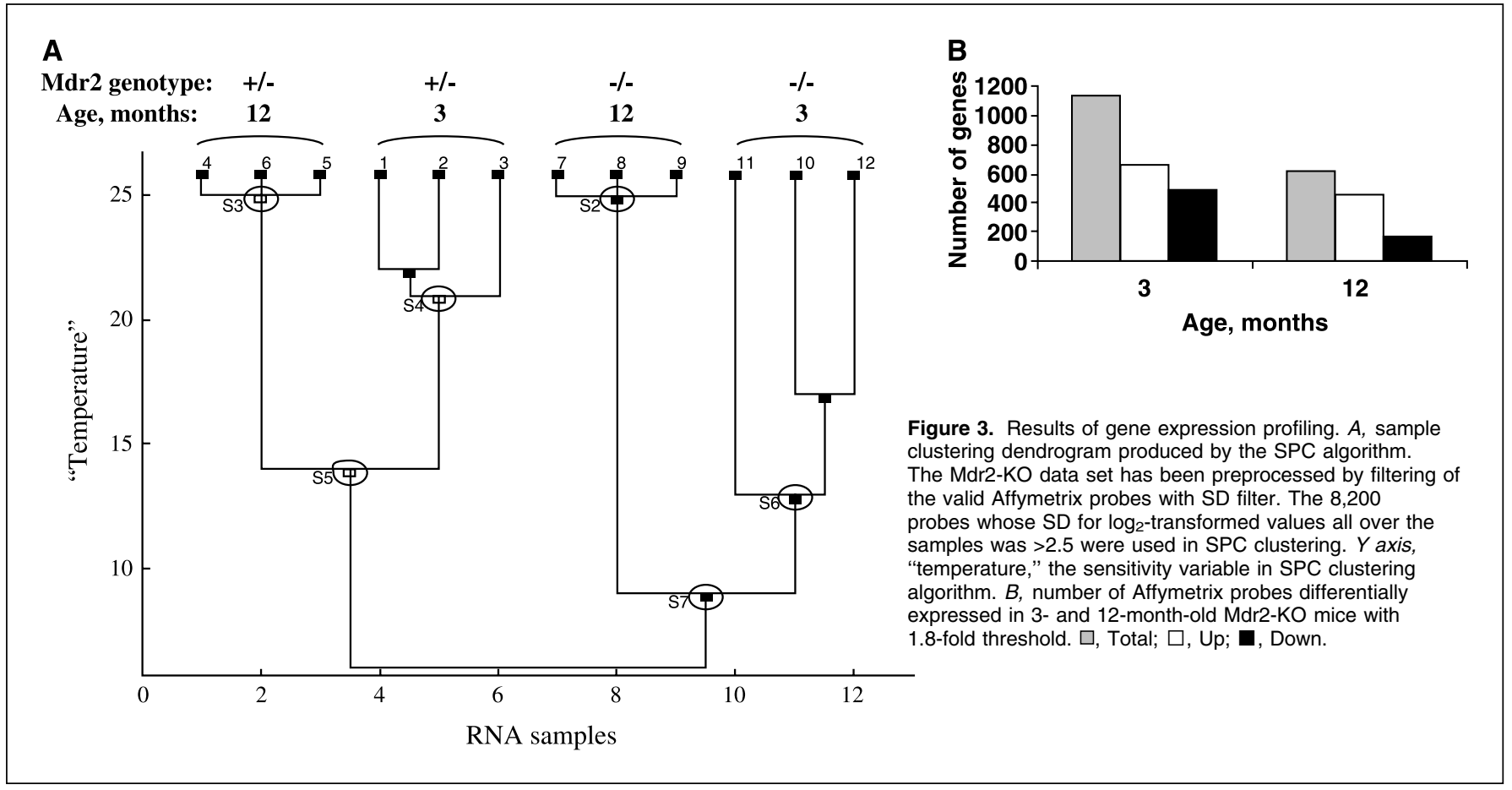

was significantly up-regulated. Phospholipase D1 enzyme may be activated by small GTPases of the ARF family. In the Mdr2-KO livers, the Arf6 gene was up-regulated especially at age 12 months (Table 2). Gene Chka, encoding a known oncogene, choline kinase A (23), was up-regulated in 12-month-old Mdr2-KO mice at mRNA level (Table 2). This was confirmed also by semiquantitative RTPCR (Supplementary Fig. S2B). Products of phosphatidylcholine degradation by phospholipases are known signaling molecules that participate in the regulation of many cellular responses; in particular, diacylglycerol activates protein kinase $\mathrm{C}$ isoforms that regulate cell cycling (24). Interestingly, gene Prkcd, encoding the $\delta$ isoform of protein kinase $\mathrm{C}$, was up-regulated in $\mathrm{Mdr2}-\mathrm{KO}$ mice at both ages (Table 2).

Markers of cell cycle and proliferation. Gene expression profiling revealed a significant group of genes controlling the process of DNA replication that were overexpressed in Mdr2-KO

Table 1. Functional groups of differentially expressed genes

Gene group

No. differentially expressed genes (3/12 mo)

\begin{tabular}{lccc} 
& & Up-regulated & Down-regulated \\
\cline { 2 - 4 } & Total & $33 / 22$ & $11 / 1$ \\
\hline Apoptosis & $44 / 23$ & $15 / 8$ & $3 / 2$ \\
Oxidative stress/antioxidant & $18 / 10$ & $67 / 38$ & $18 / 3$ \\
Immune/inflammatory response & $85 / 41$ & $15 / 13$ & $13 / 11$ \\
Oncogene & $27 / 17$ & $40 / 19$ & $3 / 0$ \\
Tumor suppressor/antioncogene & $16 / 11$ & $53 / 34$ & $13 / 2$ \\
Cell cycle & $53 / 21$ & $28 / 25$ & $23 / 13$ \\
Signal transduction & $76 / 47$ & $23 / 18$ & $8 / 3$ \\
Proteolysis & $36 / 28$ & $9 / 3$ & $3 / 0$ \\
Extracellular matrix & $26 / 18$ & $10 / 6$ & $4 / 2$ \\
Cholesterol metabolism/biosynthesis & $13 / 5$ & $21 / 20$ & $8 / 4$ \\
Fatty acid metabolism/biosynthesis & $18 / 10$ & $8 / 5$ & $13 / 5$ \\
Lipid metabolism/synthesis/transport & $34 / 25$ & $38 / 25$ & $7 / 3$ \\
Mitochondrion & $22 / 8$ & $44 / 31$ & $7 / 2$ \\
Cytoskeleton & $45 / 27$ & $442 / 290$ & \\
Cell adhesion & $51 / 35$ & & $287 / 83$ \\
Total & $729 / 373$ & & \\
& & &
\end{tabular}

NOTE: Threshold for differential expression is at least 2-fold. Gene groups were determined according to gene annotations that were downloaded by our program from the main gene databases (LocusLink, GeneAtlas, etc.). 
Table 2. Genes differentially expressed in the livers of Mdr2-KO mice at age 3 or 12 months

\begin{tabular}{|c|c|}
\hline $\begin{array}{l}\mathrm{DE} \text { at } \mathrm{DE} \text { at } \\
12 \mathrm{mo} 3 \mathrm{mo}\end{array}$ & $\begin{array}{l}\text { Gene } \\
\text { symbol }\end{array}$ \\
\hline
\end{tabular}

Functional groups

Hepatocellular carcinoma-associated expression pattern

Inflammation Oxidative Lipid/ Cell Oncogenesis
stress
$\begin{gathered}\text { phospholipid cycle } \\ \text { metabolism }\end{gathered}$

\begin{tabular}{|c|c|c|c|c|c|c|c|c|c|}
\hline 3.18 & 2.17 & ApoA4 & Apolipoprotein A-IV & & & $x$ & & & \\
\hline 2.35 & 1.97 & ApoC2 & Apolipoprotein C-II & & & $x$ & & & \\
\hline 2.69 & 1.92 & Arf6 & $A D P$-ribosylation factor 6 & & & $x$ & & $x$ & \\
\hline 4.68 & 6.65 & $A x l$ & AXL receptor tyrosine kinase & $x$ & & & & & $\times$ \\
\hline 2.16 & 1.47 & Cav1 & Caveolin, caveolae protein 1 & & & $x$ & & & \\
\hline 2.85 & 1.67 & Cav2 & Caveolin, caveolae protein 2 & & & $x$ & & & \\
\hline 2.57 & 3.50 & Ccndl & Cyclin D1 & & & & $x$ & $x$ & $\times$ \\
\hline 0.73 & 0.49 & Ccne1 & Cyclin E1 & & & & $x$ & & \\
\hline 1.95 & 1.15 & Ccng2 & Cyclin $G 2$ & & & & $x$ & & \\
\hline 6.90 & 6.47 & $C d 14$ & CD14 antigen & $x$ & & & & & \\
\hline 7.62 & 8.05 & $C d 36$ & CD36 antigen & $x$ & $\times$ & $x$ & & & $\times$ \\
\hline 1.04 & 2.13 & $C d k 4$ & Cyclin-dependent kinase 4 & & & & $x$ & & \\
\hline 4.09 & 3.22 & $C d k n 2 c$ & $\begin{array}{l}\text { Cyclin-dependent kinase } \\
\quad \text { inhibitor } 2 C(p 18, \text { Cdk4 inhibitor })\end{array}$ & & & & $\times$ & & \\
\hline 2.62 & 0.59 & Chka & Choline kinase A & & & $x$ & & $x$ & \\
\hline 0.74 & 0.46 & Chpt1 & $\begin{array}{l}\text { Choline phosphotransferase } 1 \\
\quad \text { (diacylglycerol) }\end{array}$ & & & $x$ & & & \\
\hline 2.34 & 4.49 & Copeb & $K L F 6$ & & & & $x$ & & \\
\hline 2.73 & 4.95 & Cyba & Cytochrome $b-245, \alpha$ polypeptide & $x$ & $x$ & & & & \\
\hline 2.04 & 1.53 & Ets2 & E26 avian leukemia oncogene 2 & & & & & $x$ & \\
\hline 5.25 & 1.00 & Fabp4 & aP2; fatty acid-binding protein 4 & & & $x$ & & & \\
\hline 1.85 & 1.29 & Fasn & Fatty acid synthase & & & $x$ & & & $\times$ \\
\hline 1.80 & 2.30 & Fos & FBJ osteosarcoma oncogene & & & & & $x$ & $x$ \\
\hline 1.93 & 2.38 & Gas6 & Growth arrest specific 6 & $\times$ & & & & & \\
\hline 1.91 & 2.27 & $G p x 4$ & Glutathione peroxidase 4 & & $\times$ & & & & \\
\hline 1.32 & 1.80 & Gss & Glutathione synthetase & & $\times$ & & & & \\
\hline 2.33 & 2.61 & Gsta2 & Glutathione S-transferase, $\alpha 2$ & & $\times$ & & & & \\
\hline 2.01 & 1.58 & Gsta4 & Glutathione S-transferase, $\alpha 4$ & & $\times$ & & & & \\
\hline 5.26 & 4.96 & Gstm3 & Glutathione S-transferase, $\mu 3$ & & $\times$ & & & & \\
\hline 5.80 & 4.33 & Jun & Jun oncogene & & & & & $x$ & $x$ \\
\hline 31.17 & 46.44 & Lcn2 & Lipocalin 2 & $x$ & & & & & $x$ \\
\hline 3.46 & 3.54 & Lgals1 & Lectin, galactose binding, soluble 1 & $\times$ & & & & & $\times$ \\
\hline 3.53 & 3.53 & Lgals3 & Lectin, galactose binding, soluble 3 & $x$ & & & & & $\times$ \\
\hline 4.85 & 4.60 & Lpl & Lipoprotein lipase & & & $x$ & & & \\
\hline 1.52 & 1.80 & $N f e 2 l 2$ & Nrf2 transcriptional factor & & $\times$ & & & & \\
\hline 1.67 & 2.19 & Nras & Neuroblastoma ras oncogene & & & & & $x$ & \\
\hline 1.51 & 2.81 & Pcna & Proliferating cell nuclear antigen & & & & $x$ & & \\
\hline 6.74 & 1.20 & $P d k 4$ & $\begin{array}{l}\text { Pyruvate dehydrogenase kinase, } \\
\text { isoenzyme } 4\end{array}$ & & & $x$ & & & \\
\hline 1.54 & 2.19 & Plce1 & Phospholipase $C, \epsilon 1$ & & & $x$ & & & \\
\hline 0.90 & 1.83 & Plcg2 & Phospholipase $C, \gamma 2$ & & & $x$ & & & \\
\hline 1.95 & 1.70 & Pldl & Phospholipase DI & & & $x$ & & & \\
\hline 0.55 & 1.25 & Ppara & $\begin{array}{l}\text { Peroxisome proliferator-activated } \\
\quad \text { receptor } \alpha\end{array}$ & & & $x$ & & & \\
\hline 2.00 & 1.80 & Pparg & $\begin{array}{l}\text { Peroxisome proliferator-activated } \\
\quad \text { receptor } \gamma\end{array}$ & & & $x$ & & & $x$ \\
\hline 2.36 & 2.09 & Prkcd & Protein kinase $C, \delta$ & & & $x$ & & & \\
\hline 8.76 & 4.33 & Ptgds & Prostaglandin $D_{2}$ synthase (brain) & $x$ & & $x$ & & & \\
\hline 1.98 & 3.24 & Rras & $\begin{array}{l}\text { Harvey rat sarcoma oncogene, } \\
\text { subgroup } R\end{array}$ & & & & & $\times$ & \\
\hline 1.81 & 1.40 & Rras2 & $\begin{array}{l}\text { Related Ras viral (r-ras) } \\
\quad \text { oncogene homologue } 2\end{array}$ & & & & & $x$ & \\
\hline 6.68 & 8.32 & S100a11 & S100c; calcium-binding protein A11 & & & & $x$ & & \\
\hline 3.21 & 6.06 & Saal & Serum amyloid Al & $x$ & & & & & \\
\hline
\end{tabular}


Table 2. Genes differentially expressed in the livers of Mdr2-KO mice at age 3 or 12 months (Cont'd)

\begin{tabular}{|c|c|c|c|c|c|c|}
\hline \multirow[t]{2}{*}{$\begin{array}{l}\mathrm{DE} \text { at } \\
12 \mathrm{mo}\end{array}$} & \multirow[t]{2}{*}{$\begin{array}{l}\mathrm{DE} \text { at } \\
3 \mathrm{mo}\end{array}$} & \multirow[t]{2}{*}{$\begin{array}{l}\text { Gene } \\
\text { symbol }\end{array}$} & \multirow[t]{2}{*}{ Gene name } & \multicolumn{2}{|c|}{ Functional groups } & \multirow{2}{*}{$\begin{array}{l}\text { Hepatocellular } \\
\text { carcinoma-associated } \\
\text { - expression pattern }\end{array}$} \\
\hline & & & & $\begin{array}{r}\text { Inflammation Oxidative } \\
\text { stress }\end{array}$ & $\begin{array}{l}\text { Lipid/ Cell Oncogenesis } \\
\text { phospholipid cycle } \\
\text { metabolism }\end{array}$ & \\
\hline 2.00 & 3.59 & Saa2 & Serum amyloid A2 & $x$ & & \\
\hline 2.60 & 1.42 & $S c d l$ & Stearoyl-CoA desaturase 1 & & $\times$ & $x$ \\
\hline 4.00 & 1.40 & Scd2 & Stearoyl-CoA desaturase 2 & & $x$ & \\
\hline 10.70 & 17.98 & Slpi & $\begin{array}{l}\text { Secretory leukocyte protease } \\
\quad \text { inhibitor }\end{array}$ & $\times$ & & \\
\hline 1.41 & 2.50 & Sod3 & Superoxide dismutase 3 , extracellular & $\times$ & & \\
\hline 1.90 & 2.20 & Sppl & Osteopontin, secreted phosphoprotein 1 & $\times$ & & $\times$ \\
\hline 1.81 & 3.93 & Stat1 & $\begin{array}{l}\text { Signal transducer and activator } \\
\text { of transcription } 1\end{array}$ & $\times$ & & \\
\hline
\end{tabular}

NOTE: Listed are genes that had differential expression (DE) at least 1.8-fold (mean of Mdr2-KO mice relative to the mean of control Mdr2heterozygotes) at age 3 or 12 months.

mice mainly at age 3 months (Table 2; Supplementary Table S3). This group included six minichromosome maintenance proteins responsible for "chromatin licensing" (25), cyclin-dependent kinase 4 (Cdk4), and proliferating cell nuclear antigen (PCNA), a known proliferation marker. Immunohistochemical staining confirmed that PCNA was significantly up-regulated in the livers of 3-month-old Mdr2-KO mice (Supplementary Fig. S4B compared with Supplementary Fig. S4A) but only slightly up-regulated in 12-month-old mutants (Supplementary Fig. S4D compared with Supplementary Fig. S4C). PCNA was mostly detected in hepatocytes, showing that DNA replication takes place mainly in Mdr2-KO hepatocytes. However, when hepatocyte mitoses were directly counted on histologic sections, significant hepatocyte proliferation was detected only in 9- and 12-month-old Mdr2-KO mice (Fig. 1C). Increased DNA replication without hepatocyte mitoses in 3-month-old Mdr2-KO mice could be explained by hepatocyte cell cycle arrest. A possible regulator responsible for this effect is nuclear phosphoprotein S100A11/S100c (26) that was significantly overexpressed in Mdr2-KO livers at both ages 3 and 12 months (Table 2).

Expression of cyclin D1, a key regulator of cell cycle progression and a known oncogene, was significantly increased at mRNA level in the livers of both 3- and 12-month-old Mdr2-KO mice (Table 2). Overexpression of cyclin D1 protein was also confirmed by Western blot analysis of liver extracts from Mdr2-KO and control mice (Fig. $4 D$ ). Two inhibitors of the cyclin D1-Cdk4/6 complex activity were overexpressed at mRNA level in Mdr2-KO livers at both ages (Table 2): p18Ink4c, encoded by the gene $C d k n 2 c$, and KLF6, encoded by the gene Copeb (27).

These results suggest that hepatocyte replication in Mdr2-KO mice at an early stage of liver disease is blocked by multiple protective mechanisms. However, between ages 6 and 9 months, these protective mechanisms fail, resulting in intensive hepatocyte proliferation leading to the development of multiple dysplastic nodules by age 12 months.

Oncogenes and tumor suppressors. Gene expression profiling revealed a significant number of oncogenes and tumor suppressors that were differentially expressed (mainly up-regulated) in Mdr2-KO mice at both ages (Table 1). The most prominent was the Ras family of proteins: Nras, Rras, and Rras2 (Table 2) and Rhob, Rhoc, Rhod, and Rhoe (Supplementary Table S3). Genes Lgals1 and Lgals3, encoding known activators of Ras signaling, galectin-1 and galectin-3, which augment activation of H-Ras and K-Ras, respectively $(28,29)$, were also overexpressed in Mdr2-KO mice at both ages (Table 2). Thus, we tested the levels of Ras proteins and activated Ras (Ras-GTP complexes) in mouse liver extracts by immunoblotting with Pan-Ras antibody (detects H-Ras, N-Ras, and K-Ras) or with antiR-Ras antibody (detects R-Ras only). In a pull-down assay of activated Ras proteins, the detection was done only with Pan-Ras antibody.

As can be seen in Fig. $4 D$, the average level of the Ras isoforms detected by the Pan-Ras antibody was higher in Mdr2-KO mice at both ages. However, levels of the activated Ras in Mdr2-KO mice were not higher than those in controls at any age: at age 12 months, they were comparable, whereas at age 3 months they varied from equal to significantly lower than in controls (seven 3-month-old Mdr2-KO mice produced similar results). Thus, results inferred partial suppression of Ras activation in 3-month-old Mdr2$\mathrm{KO}$ mice and restoration of Ras activation to normal levels by age 12 months. Levels of the R-Ras protein in Mdr2-KO livers were significantly lower than in controls at both ages despite confirmation of the up-regulation of R-Ras mRNA by semiquantitative RT-PCR at both ages 3 and 12 months (data for 3-monthold mice are shown in Supplementary Fig. S2A).

Up-regulation of genes Jun and Fos, encoding two components of the transcription factor activator protein-1 (AP-1), c-Jun and c-Fos, was detected in Mdr2-KO mice at both ages by microarray (Table 2) and by RT-PCR (Supplementary Fig. S2) techniques. The critical role of the c-Jun proto-oncoprotein in hepatocellular carcinoma development was recently shown on a mouse model of chemically induced hepatocarcinogenesis (30). On the other hand, its functional antagonist, transcription factor KLF6, encoded by the gene Copeb (31), was also found to be up-regulated in Mdr2KO mice at both ages (Table 2).

\section{Discussion}

In the present study, we did detailed gene expression, histologic, and biochemical analyses to identify potentially important regulators that function at the early stages of hepatocellular 
A

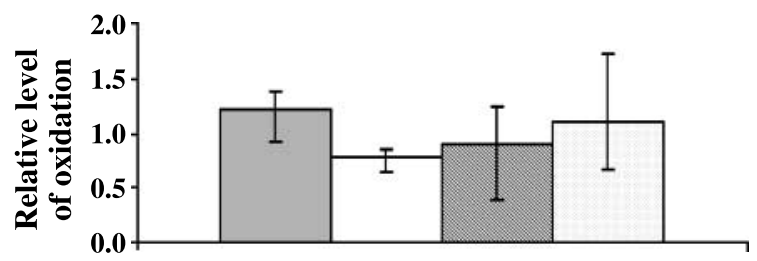

B

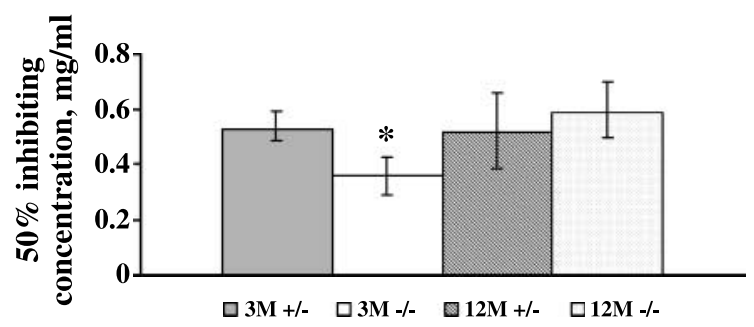

C

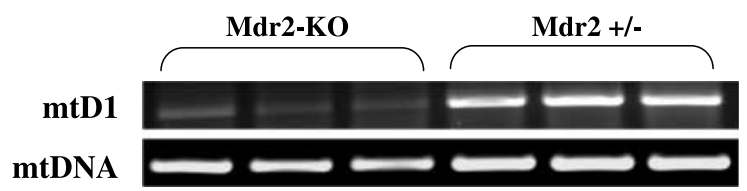

D

3 months

12 months

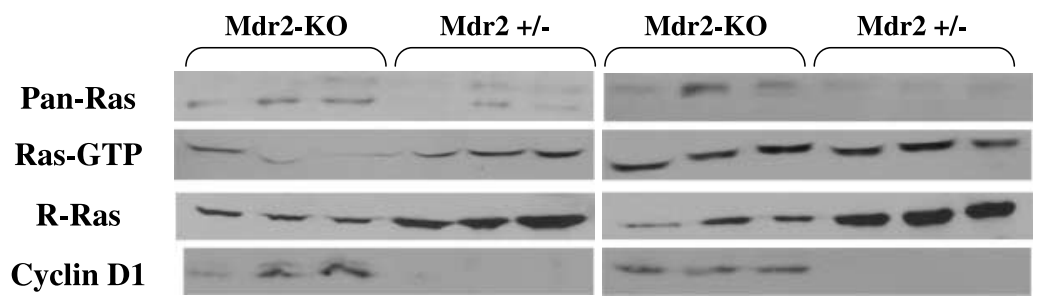

Figure 4. Analysis of the liver tissues of Mdr2-KO and control Mdr2(+/-) mice. $A$, mean relative levels of protein oxidation in different experimental groups (three males in each group) measured with the Oxyblot kit as described in Materials and Methods. $B$, concentrations of liver tissue extracts $(\mathrm{mg} / \mathrm{mL}$; mean of three males per each experimental group) producing $50 \%$ inhibition of AAPH-induced luminol-amplified chemiluminescence (as described in Materials and Methods). The total antioxidant capacity of liver tissue is inversely proportional to the $\mathrm{IC}_{50}$ of liver extract. *, $P<0.05$, compared with age-matched heterozygous control. $C$, detection of the deletions in mtDNA by semiquantitative RT-PCR in 12-month-old mice (three males per each group). $m t D 1$, DNA fragment corresponding to the unstable region of mtDNA that is frequently deleted in aging or at oxidative stress; $m t D N A$, DNA

fragment corresponding to a stable region of mtDNA. $D$, immunoblot analysis of liver tissue extracts from 3-month-old (left) or 12-month-old (right) Mdr2-KO and control heterozygous mice. Pan-Ras antibody revealed two main bands in immunoblots of liver extracts [ $\sim 21$ (bottom) and 24 (top) kDa]. At age 3 months, Ras was detected mostly in the bottom band, whereas at age 12 months Ras was detected mostly in the top band. Ras-GTP complexes were immunoprecipitated with GST-Raf1-RBD as described in Materials and Methods and immunoblotted with Pan-Ras antibody. carcinoma development in Mdr2-KO mice. Our results show that liver disease in Mdr2-KO mice at the precancerous stage is characterized by a cross-talk between regulatory systems that control immune response, oxidative stress, hepatocyte proliferation, and lipid metabolism. These data suggest that multiple adaptive mechanisms have been induced in each of these systems to cope with primary genetic defect and its physiologic consequences in this hepatocellular carcinoma model.

One of the central issues for this model is the role of the immune response in hepatocarcinogenesis. Recently, in this model, the important role of TNF- $\alpha$ and NF- $\kappa \mathrm{B}$ in promoting hepatocellular carcinoma development was shown (32). Here, we describe massive infiltration of monocytes/macrophages and neutrophils in the portal tracts and liver parenchyma of 3-month-old Mdr2-KO mice, which was reduced but was still present at age 12 months. However, mRNA of inducible nitric oxide synthase was not up-regulated at both ages, indicating possible suppression of macrophage activation. Several genes whose products may accomplish this protective function were found to be up-regulated in the livers of both 3- and 12-month-old Mdr2-KO mice: Axl, Gas6, Lgals1, Pparg, Slpi, and Spp1 (11-15). Similarly, mRNA of TNF- $\alpha$ and serum amyloid proteins, A1 and A2, were overexpressed in the livers of Mdr2-KO mice at both ages (Supplementary Fig. S2; Table 2); these factors may activate NF- $\kappa$ B in epithelial cells $(10,32)$, a response that may protect mature hepatocytes against immune attack and biological stress (33). However, many of these gene products that are potentially protective against inflammation could also promote hepatocarcinogenesis $(11,34)$. The antiapoptotic effect of NF- $\kappa$ B on hepatocytes has been shown in the Mdr2-KO model (32). However, in a chemically induced liver cancer model, it was recently shown that NF- $\kappa$ B induction in hepatocytes is antitumorigenic, whereas in Kupffer cells it is procarcinogenic (35). Thus, the actual role of NF- $\kappa \mathrm{B}$ activation in hepatocarcinogenesis in this hepatocellular carcinoma model requires further elucidation.

Oxidative stress has long been implicated in carcinogenesis. It is known that reactive oxygen species activate signal transduction pathways, including $\mathrm{AP}-1$ and $\mathrm{NF}-\kappa \mathrm{B}$, and cause oxidative DNA damage, increasing risk of cancer development (36). We could not detect signs of increased oxidation of total liver protein or of mtDNA in Mdr2-KO mice. However, up-regulation of the Cyba gene encoding the critical component of the membrane-bound oxidase of phagocytes that generates superoxide (Table 2) indicates that reactive oxygen species are produced in the inflamed livers of Mdr2-KO mice at both studied ages.

Results of this study revealed a significant number of dysplastic nodules in the livers of 12-month-old Mdr2-KO mice. We suggest that both intensive hepatocyte death and compensating proliferation take place in their livers. This death may result either from toxic 
effect of bile acid leakage (3) or from a nondirect immune-mediated hepatocyte injury (e.g., reactive oxygen species produced by infiltrating phagocytes) or due to overrun of cell protective mechanisms after age 6 months. The increased hepatocyte proliferation may elevate hepatocellular carcinoma incidence by accumulation of spontaneous mutations during normal DNA replication (37).

In the present work, one of the most prominent features discovered in the Mdr2-KO mice is overexpression in their livers of cyclin D1, a known oncogene that is also overexpressed in the early stages of many types of human tumors (38). In many cancer types, including hepatocellular carcinoma, cyclin D1 overexpression is associated with poor prognosis (39). Supporting its critical role in hepatocarcinogenesis, transgenic mice that overexpress cyclin D1, specifically in the liver, developed hepatocellular carcinoma (40), whereas antisense oligonucleotides to cyclin D1 inhibited growth of hepatoma cells (41). Up-regulation of c-Jun in the Mdr2-KO livers that was shown in our laboratory by both microarray and RT-PCR and earlier by immunohistochemistry (32) is of significance to liver cancer development not only due to up-regulation of cyclin D1 by cJun but also because c-Jun itself promotes liver cancer in mice by antagonizing p53 activity (30). Supporting the increased activity of $\mathrm{c}$-Jun is the recent finding that livers of Mdr2-KO mice overexpress the c-Jun $\mathrm{NH}_{2}$-terminal kinase (32), which is responsible for cyclin D1 expression and hepatocyte proliferation during liver regeneration.

One of the most striking features of liver disease in Mdr2-KO mice is slowly developing liver steatosis. Analysis of underlying molecular mechanisms suggests a key role of PPAR- $\gamma$ in this process. Although the Pparg gene was overexpressed in Mdr2-KO mice at both ages, dynamics of expression of its target genes showed that it was activated preferentially at age 12 months. This correlates with the increased overexpression at this age of prostaglandin synthase $D_{2}$ (gene Ptgds) that should produce increased levels of prostaglandin $15 \mathrm{~d}-\mathrm{PGJ}_{2}$, a natural PPAR- $\gamma$ ligand. Up-regulation of adipocytespecific PPAR- $\gamma$-regulated genes (e.g., Fabp4) shows that steatosis developed in Mdr2-KO mice may be characterized as "adipogenic hepatic steatosis" or "hepatic adiposis" (19).

Besides its role in lipid metabolism, PPAR- $\gamma$ is also involved in the regulation of processes important for cancer development: response to oxidative stress (42), inflammation (43), and regulation of cell cycle (44). Its target gene, $C d 36$, links lipid metabolism to oxidative stress (45); it was strongly up-regulated in the livers of Mdr2-KO mice at both ages (Table 2).

Our results revealed up-regulation in Mdr2-KO mice of mRNA of the enzymes metabolizing phosphatidylcholine: phospholipase $\mathrm{C}$ at 3 months and phospholipase D1 and choline kinase A at 12 months. These changes may have a profound effect on hepatocarcinogenesis. Activation of choline kinase $\mathrm{A}$ has been recently found to be a critical factor for mammary epithelial cell proliferation and breast tumor progression (23). Among all oncogenes differentially expressed in the liver of Mdr2-KO mice, choline kinase A was the only one exclusively up-regulated at age 12 months. This expression pattern suggests that choline kinase A may play an important role in hepatocarcinogenesis in Mdr2-KO mice. The products of phospha- tidylcholine degradation by phospholipases D and C, phosphatidic acid and diacylglycerol, act as second messengers essential for mitogenic activity of growth factors. It is known that phosphatidylcholine hydrolysis may activate the Ras-Rafl-mitogen-activated protein kinase (MAPK) cascade downstream of Ras but upstream of Rafl (46). Diacylglycerol may activate protein kinase C (24). Protein kinase $\mathrm{C} \delta$, which was up-regulated in Mdr2-KO mice at both ages, is capable of both positive and negative regulation of cell proliferation and may be activated by TNF- $\alpha$ and oxidative stress $(24,47)$. Although we found no evidence of Ras activation in the livers of Mdr2-KO mice at both ages, the Ras-Rafl-MAPK signaling pathway may be activated in this model by products of phosphatidylcholine metabolism and by TNF- $\alpha$ downstream of Ras. Interestingly, similar phenotypes and mechanisms characterize hepatocarcinogenesis in rats supplied with choline-deficient diet: fatty liver, increased levels of phosphatidylcholine metabolism, sustained activation of protein kinase $C$, and increased level of protein kinase $C \delta(48,49)$. However, in contrast to the Mdr2-KO model, the choline-deficient diet is effective only for males in both rats and mice.

In conclusion, we found induction of multiple adaptive mechanisms to chronic liver disease in Mdr2-KO mice in both early and late precancerous stages, which could protect the liver from inflammation, oxidative stress, and uncontrolled hepatocyte proliferation. However, prolonged overexpression of these mechanisms could have an effect on liver cancer development in these mice. We have identified significant disturbances in lipid and phosphatidylcholine metabolism that may cause activation of oncogenic pathways. Finally, we have shown overexpression in the livers of Mdr2-KO mice of cyclin D1 protein, a key oncogene, which may be a good candidate for functional $\mathrm{KO}$ experiments in this hepatocellular carcinoma model. It should be mentioned that the use of whole liver extracts for gene expression profiling is only the first step in determining the roles of the differentially expressed genes discussed above in hepatocellular carcinoma development. More detailed knowledge concerning the role of these genes in hepatocarcinogenesis in Mdr2-KO mice should be further elucidated by immunohistochemical determination of cell type specificity, cytoplasmic/nuclear localization, and local expression pattern of the appropriate proteins and by comparative gene expression profiling of tumorous versus nontumorous liver tissues.

\section{Acknowledgments}

Received 8/17/2005; revised 1/12/2006; accepted 2/8/2006.

Grant support: Salzberg Foundation and Kamea Scientific Foundation of the Israeli Government (D. Goldenberg), Israeli Science Ministry grant (Gene Therapy Strategic Center), Blum Foundation, Greenspoon Foundation, Horwitz Foundation (E. Galun), and Horwitz Foundation through The Center for Complexity Science (M. Katzenellenbogen and H. Barash).

The costs of publication of this article were defrayed in part by the payment of page charges. This article must therefore be hereby marked advertisement in accordance with 18 U.S.C. Section 1734 solely to indicate this fact.

We thank Dr. Eli Pikarsky (Department of Pathology, Hadassah University Hospital), Dr. Amnon Peled (Goldyne Savad Institute of Gene Therapy, Hadassah University Hospital), and Drs. Yoel Kloog and Roni Haklai (Department of Neurobiochemistry, Tel-Aviv University) for their support and useful discussions, Ariel Israel for assisting in gene expression profiling analysis, and Carol Levy and Esther Korzin-Bez for assistance in their work with mice.

\section{References}

1. Lee JS, Chu IS, Mikaelyan A, et al. Application of comparative functional genomics to identify best-fit mouse models to study human cancer. Nat Genet 2004; 36:1306-11.

2. Smit JJ, Schinkel AH, Oude Elferink RP, et al. Homozygous disruption of the murine mdr2 P-glycopro- tein gene leads to a complete absence of phospholipid from bile and to liver disease. Cell 1993;75:451-62.

3. Fickert P, Fuchsbichler A, Wagner M, et al. Regurgitation of bile acids from leaky bile ducts causes 
sclerosing cholangitis in Mdr2 (Abcb4) knockout mice. Gastroenterology 2004;127:261-74.

4. Mauad TH, van Nieuwkerk CM, Dingemans KP, et al. Mice with homozygous disruption of the mdr2 P-glycoprotein gene. A novel animal model for studies of nonsuppurative inflammatory cholangitis and hepatocarcinogenesis. Am J Pathol 1994; 145:1237-45.

5. Blatt M, Wiseman S, Domany E. Superparamagnetic clustering of data. Phys Rev Lett 1996;76:3251-4.

6. Tanhauser SM, Laipis PJ. Multiple deletions are detectable in mitochondrial DNA of aging mice. J Bio Chem 1995;270:24769-75.

7. Kawagoe M, Nakagawa K. Attenuation of luminolamplified chemiluminescent intensity and lipid peroxidation in the livers of quercetin-fed mice. Toxicol Lett 2000;114:189-96.

8. Getz G, Levine E, Domany E. Coupled two-way clustering analysis of gene microarray data. Proc Nat Acad Sci U S A 2000;97:12079-84.

9. Hatanaka E, Furlaneto CJ, Ribeiro FP, Souza GM, Campa A. Serum amyloid A-induced mRNA expression and release of tumor necrosis factor- $\alpha$ (TNF- $\alpha$ ) in human neutrophils. Immunol Lett 2004;91:33-7.

10. Jijon HB, Madsen KL, Walker JW, Allard B, Jobin C. Serum amyloid A activates NF- $\kappa \mathrm{B}$ and proinflammatory gene expression in human and murine intestinal epithelial cells. Eur J Immunol 2005;35:718-26.

11. Liu FT, Rabinovich GA. Galectins as modulators of tumour progression. Nat Rev Cancer 2005;5:29-41.

12. Lemke G, Lu Q. Macrophage regulation by Tyro 3 family receptors. Curr Opin Immunol 2003;15:31-6.

13. Ricote M, Li AC, Willson TM, Kelly CJ, Glass CK. The peroxisome proliferator-activated receptor- $\gamma$ is a negative regulator of macrophage activation. Nature 1998; 391:79-82.

14. Guo H, Cai CQ, Schroeder RA, Kuo PC. Osteopontin is a negative feedback regulator of nitric oxide synthesis in murine macrophages. J Immunol 2001;166: 1079-86.

15. Greene CM, McElvaney NG, O’Neill SJ, Taggart CC. Secretory leucoprotease inhibitor impairs Toll-like receptor 2- and 4-mediated responses in monocytic cells. Infect Immun 2004;72:3684-7.

16. Factor VM, Laskowska D, Jensen MR, Woitach JT, Popescu NC, Thorgeirsson SS. Vitamin E reduce chromosomal damage and inhibits hepatic tumo formation in a transgenic mouse model. Proc Natl Acad Sci U S A 2000;97:2196-201.

17. Kohen R, Vellaichamy E, Hrbac J, Gati I, Tirosh O Quantification of the overall reactive oxygen species scavenging capacity of biological fluids and tissues. Free Radic Biol Med 2000;28:871-9.

18. Scher JU, Pillinger MH. $15 \mathrm{~d}-\mathrm{PGJ}_{2}$ : the anti-inflammatory prostaglandin? Clin Immunol 2005;114:100-9. 19. Yu S, Matsusue $K$, Kashireddy P, et al. Adipocyte specific gene expression and adipogenic steatosis in the mouse liver due to peroxisome proliferator-activated receptor $\gamma 1$ (PPAR $\gamma 1)$ overexpression. J Biol Chem 2003; 278:498-505.

20. Gavrilova O, Haluzik M, Matsusue K, et al. Live peroxisome proliferator-activated receptor $\gamma$ contributes to hepatic steatosis, triglyceride clearance, and regulation of body fat mass. J Biol Chem 2003;278 34268-76.

21. Matsusue K, Haluzik M, Lambert G, et al. Liverspecific disruption of PPAR $\gamma$ in leptin-deficient mice improves fatty liver but aggravates diabetic phenotypes. J Clin Invest 2003;111:737-47.

22. Merkel M, Eckel RH, Goldberg IJ. Lipoprotein lipase: genetics, lipid uptake, and regulation. J Lipid Res 2002, 43:1997-2006.

23. de Molina AR, Banez-Coronel M, Gutierrez R, et al. Choline kinase activation is a critical requirement for the proliferation of primary human mammary epithelial cells and breast tumor progression. Cancer Res 2004;64: 6732-9.

24. Nishizuka Y. The protein kinase $\mathrm{C}$ family and lipid mediators for transmembrane signaling and cell regulation. Alcohol Clin Exp Res 2001;25:3-7S

25. Tachibana KE, Gonzalez MA, Coleman N. Cell-cycledependent regulation of DNA replication and its relevance to cancer pathology. J Pathol 2005;205:123-9. 26. Sakaguchi M, Miyazaki M, Inoue Y, et al. Relation ship between contact inhibition and intranuclear S100C of normal human fibroblasts. J Cell Biol 2000; 149:1193-206.

27. Benzeno S, Narla G, Allina J, et al. Cyclin-dependen kinase inhibition by the KLF6 tumor suppressor protein through interaction with cyclin D1. Cancer Res 2004;64: 3885-91.

28. Elad-Sfadia G, Haklai R, Ballan E, Gabius HJ, Kloog Y. Galectin-1 augments Ras activation and diverts Ras signals to Raf- 1 at the expense of phosphoinositide 3 kinase. J Biol Chem 2002;277:37169-75.

29. Elad-Sfadia G, Haklai R, Balan E, Kloog Y. Galectin-3 augments K-Ras activation and triggers a Ras signal that attenuates ERK but not phosphoinositide 3-kinase activity. J Biol Chem 2004;279:34922-30.

30. Eferl R, Ricci R, Kenner L, et al. Liver tumor development: c-Jun antagonizes the proapoptotic activity of p53. Cell 2003;112:181-92.

31. Slavin DA, Koritschoner NP, Prieto CC, Lopez-Diaz FJ, Chatton B, Bocco JL. A new role for the Kruppel-like transcription factor KLF6 as an inhibitor of c-Jun protooncoprotein function. Oncogene 2004;23:8196-205.

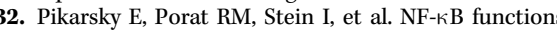
as a tumour promoter in inflammation-associated cancer. Nature 2004;431:461-6.

33. Lavon I, Pikarsky E, Gutkovich E, et al. Nuclea factor $-\kappa \mathrm{B}$ protects the liver against genotoxic stress and functions independently of p53. Cancer Res 2003; 63:25-30.

34. Devoogdt N, Revets H, Ghassabeh GH, De Baetselier P. Secretory leukocyte protease inhibitor in cancer development. Ann N Y Acad Sci 2004;1028:380-9.
35. Maeda S, Kamata H, Luo JL, Leffert H, Karin M. IKK $\beta$ couples hepatocyte death to cytokine-driven compensatory proliferation that promotes chemical hepatocarcinogenesis. Cell 2005;121:977-90.

36. Klaunig JE, Kamendulis LM. The role of oxidative stress in carcinogenesis. Annu Rev Pharmacol Toxicol 2004;44:239-67.

37. Cohen SM, Ellwein LB. Genetic errors, cell proliferation, and carcinogenesis. Cancer Res 1991;51:6493-505. 38. Weinstein IB. Relevance of cyclin D1 and other molecular markers to cancer chemoprevention. J Cell Biochem Suppl 1996;25:23-8.

39. Nishida N, Fukuda Y, Komeda T, et al. Amplification and overexpression of the cyclin D1 gene in aggressive human hepatocellular carcinoma. Cancer Res 1994;54: 3107-10.

40. Deane NG, Parker MA, Aramandla R, et al. Hepatocellular carcinoma results from chronic cyclin D1 overexpression in transgenic mice. Cancer Res 2001;61: 5389-95.

41. Uto $\mathrm{H}$, Ido A, Moriuchi A, et al. Transduction of antisense cyclin D1 using two-step gene transfer inhibits the growth of rat hepatoma cells. Cancer Res 2001;61: 4779-83.

42. Park EY, Cho IJ, Kim SG. Transactivation of the PPAR-responsive enhancer module in chemopreventive glutathione $S$-transferase gene by the peroxisome proliferator-activated receptor- $\gamma$ and retinoid $X$ receptor heterodimer. Cancer Res 2004;64:3701-13.

43. Dubuquoy L, Dharancy S, Nutten S, Pettersson S, Auwerx J, Desreumaux P. Role of peroxisome proliferator-activated receptor $\gamma$ and retinoid $X$ receptor heterodimer in hepatogastroenterological diseases. Lancet 2002;360:1410-8.

44. Qin C, Burghardt R, Smith R, Wormke M, Stewart J, Safe S. Peroxisome proliferator-activated receptor $\gamma$ agonists induce proteasome-dependent degradation of cyclin D1 and estrogen receptor $\alpha$ in MCF-7 breast cancer cells. Cancer Res 2003;63:958-64.

45. Fuhrman B, Volkova N, Aviram M. Oxidative stress increases the expression of the CD36 scavenger receptor and the cellular uptake of oxidized low-density lipoprotein in macrophages from atherosclerotic mice: protective role of antioxidants and of paraoxonase. Atherosclerosis 2002;161:307-16.

46. Cai H, Erhardt P, Troppmair J, et al. Hydrolysis of phosphatidylcholine couples Ras to activation of Raf protein kinase during mitogenic signal transduction. Mol Cell Biol 1993;13:7645-51.

47. Jackson DN, Foster DA. The enigmatic protein kinase Co: complex roles in cell proliferation and survival. FASEB J 2004;18:627-36.

48. Ghoshal AK, Farber E. The induction of liver cancer by dietary deficiency of choline and methionine without added carcinogens. Carcinogenesis 1984;5:1367-70.

49. Zeisel SH, da Costa KA, Albright CD, Shin $\mathrm{OH}$ Choline and hepatocarcinogenesis in the rat. Adv Exp Med Biol 1995;375:65-74. 